\title{
Extending practical flow chemistry into the undergraduate curriculum via the use of a portable low-cost 3D printed continuous flow system
}

\author{
Matthew R. Penny ${ }^{1}$ (D) Natalie Tsui $^{1} \cdot$ Stephen T. Hilton ${ }^{1}$ (D) \\ Received: 28 September 2020 / Revised: 21 October 2020 / Accepted: 22 October 2020 / Published online: 11 November 2020 \\ (C) The Author(s) 2020
}

\begin{abstract}
Continuous flow chemistry is undergoing rapid growth and adoption within the pharmaceutical industry due to its ability to rapidly translate chemical discoveries from medicinal chemistry laboratories into process laboratories. Its growing significance means that it is imperative that flow chemistry is taught and experienced by both undergraduate and postgraduate synthetic chemists. However, whilst flow chemistry has been incorporated by industry, there remains a distinct lack of practical training and knowledge at both undergraduate and postgraduate levels. A key challenge associated with its implementation is the high cost $(>\$ 25,000)$ of the system's themselves, which is far beyond the financial reach of most universities and research groups, meaning that this key technology remains open to only a few groups and that its associated training remains a theoretical rather than a practical subject. In order to increase access to flow chemistry, we sought to design and develop a small-footprint, low-cost and portable continuous flow system that could be used to teach flow chemistry, but that could also be used by research groups looking to transition to continuous flow chemistry. A key element of its utility focusses on its 3D printed nature, as low-cost reactors could be readily incorporated and modified to suit differing needs and educational requirements. In this paper, we demonstrate the system's flexibility using reactors and mixing chips designed and 3D printed by an undergraduate project student (N.T.) and show how the flexibility of the system allows the investigation of differing flow paths on the same continuous flow system in parallel.
\end{abstract}

Keywords 3D Printing $\cdot$ Additive Manufacturing $\cdot$ Flow Chemistry $\cdot$ Reactionware $\cdot$ Fluidics

\section{Introduction}

Despite the fact that there are a rapidly increasing number of publications concerning continuous flow chemistry and that

\section{Highlights}

- We demonstrate the small-footprint nature of our continuous flow system that is suitable for undergraduate education.

- We demonstrate that it can be used with a variety of 3D printed mixing chips to illustrate mixing and the associated methodology.

- We show how the flexibility of the system allows for parallel reaction optimisation via residence time or reactor design on the same smallfootprint continuous flow system.

Supplementary Information The online version contains supplementary material available at https://doi.org/10.1007/s41981-02000122-5.

Stephen T. Hilton

s.hilton@ucl.ac.uk

1 UCL School of Pharmacy, 29-39 Brunswick Square, WC1N 1AX London, UK the adoption of continuous flow chemistry by industry has been encouraged by both the Federal Drug Agency (FDA) and the Chinese government [1] it is surprising that the subject receives little or no attention within the training of synthetic chemists at an undergraduate level $[2,3]$. This deficit often continues into research groups, with postgraduate students also not receiving exposure to continuous flow synthesis at both $\mathrm{PhD}$ and postgraduate levels. This growing imbalance between the skills and training that undergraduates and postgraduates receive, versus the continuous flow chemistry requirements of those of the pharmaceutical industry needs urgent rectification if universities and research groups are to equip the next generation of chemists with the skills and required experience for the pharmaceutical industry. Additionally, if flow chemistry is to reach a stage where adoption is not limited only to "early adopters" and becomes concentrated in a few research groups with the financial resources to incorporate it into their research, then the early introduction of flow chemistry in undergraduate practical courses is of paramount importance. Unfortunately, the high costs of 
commercial equipment needed for flow chemistry makes this difficult for many universities due to a large capital expenditure requirement. Even if universities have the financial resources to purchase systems, these are often limited to one or two as the high costs ( $>\$ 25,000$ per system) means that it is fanciful to imagine a case where a university is able to provide access for one system per student pair.

As such, in those Universities incorporating flow chemistry into the curriculum, current "best practice" for undergraduate flow chemistry education focuses on the use of syringe pumps, due to the fact that these are straightforward to use and can be easily distributed along with inexpensive pieces of tubing and connectors [4]. However, costs can easily mount up if teaching to a large class $(>50)$ at the same time. In addition, there is a practical challenge of using syringe pumps, as they are relatively large and take up a large space in a fume cupboard or laboratory bench, meaning that only larger groupings of students can use these, rather than the more ideal use of a system by a pair of students. A growing alternative to the use of expensive syringe pumps focuses on a "DIY" approach to the construction of flow equipment via the $3 \mathrm{D}$ printing of syringe pumps controlled via microcontrollers, but this often leads to widespread differences in practice between universities and research groups and a plethora of systems [5].

As a result of these challenges, along with the growing 3D printing research in our group in both batch and flow, we set out to develop an adaptable, portable, modular, small footprint, low-cost 3D printed continuous flow system that could incorporate a number of flow paths as per user requirements that could be used by undergraduates in practical classes and also by research groups which was described recently [6-9] (Fig. 1).

The system itself was designed to be a pressure driven one where the normal geared syringe pumps or HPLC based pumps were replaced with a compressed gas source of fluidic flow, where the rate could be readily predicted and calculated based on the pressure, solvent viscosity, ambient temperature and dimensions of a variable restrictor capillary placed at the end of the flow path, according to the Hagen-Poiseuille equation [6]. Due to the high resistance through the capillary, the temperature of the reaction and the architecture of the flow path through mesoscale reactors can be readily altered without affecting the flow rate.
In this research, we describe how we sought to try to understand the potential of the system and how it could be used to flexibly teach undergraduate students continuous flow chemistry to illustrate the concept of flow and to allow for reaction analysis from the flow reactor. The student was a masters of Pharmacy project student (N.T.), which made it more challenging as the level of exposure to synthetic chemistry is lower in Pharmacy than in chemistry as a discipline.

As such, rather than burden the student with theoretical explanations behind the setup of the flow equipment, it was decided that they would be involved in the assembly of the modules to help understand how the equipment works and to visualise the flow path. The student would also be able to get an appreciation of the key components required for flow chemistry, namely: a method for pumping fluid, a mixing device, a reaction stage and a back-pressure regulator [10]. In order to save time, the 3D printed parts were prepared in advance. However, the student would gain experience by later designing and 3D printing reactors and mixing devices. Due to the short time available for the project (4 months), we needed to be able to put together the equipment required and disassemble it in a short amount of time. The four modules were stacked on to a magnetic stirrer hotplate which meant that minimal additional space was required when compared to batch reactions (Fig. 2). An additional pedagogical benefit to the student of putting together flow equipment themselves is the ability to visualise the flow path and understand important concepts such as laminar flow, dispersion and residence time as well as the benefits of fine heat control and space integration of multi-step syntheses [11].

\section{Results and discussion}

\section{Preliminary mixing experiments}

In order to introduce the student to the concept of continuous flow synthesis and its focus on the efficient and timely mixing of reagents, we decided to design a series of experiments that focussed on the mixing of two alcohols ( $p$-cresol and 2chlorophenol) via separate injection at injection loop $\mathrm{A}$ and injection loop B of our system at a low flow rate $(0.17 \mathrm{~mL} /$
Fig. 1 a The structure of our lowcost continuous flow system designed to fit existing stirrer hotplates; $\mathbf{b}$ The Assembled System; c The modular nature means that it is portable and easily fits within a storage briefcase
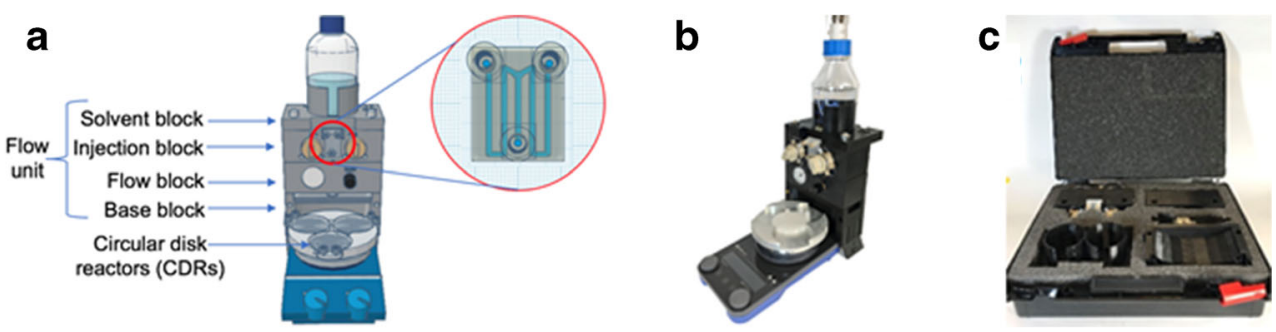


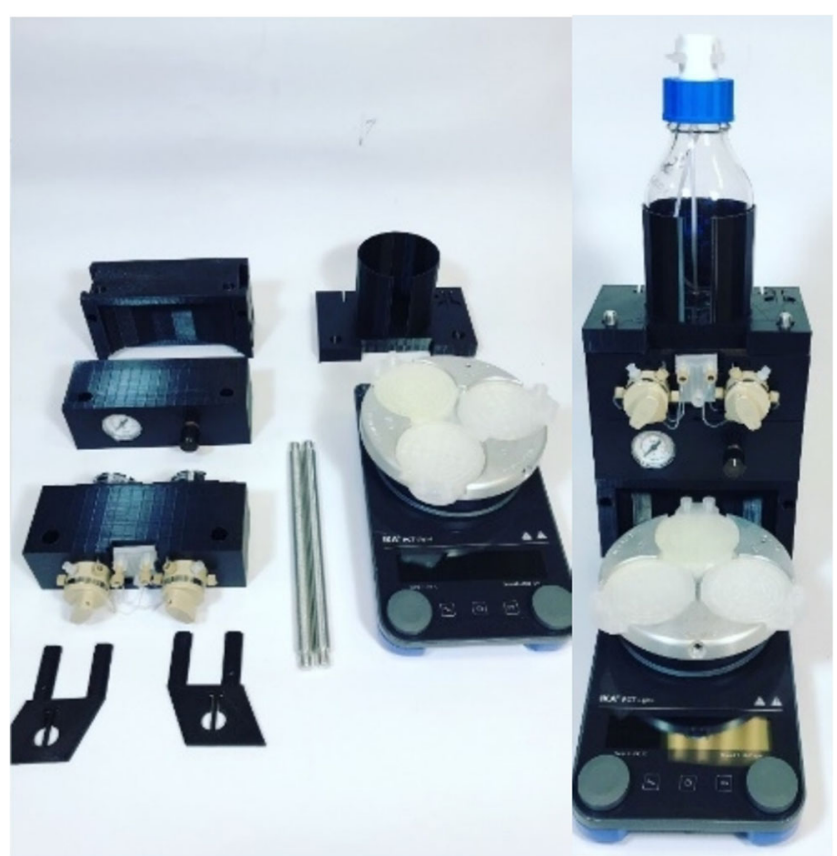

Fig. 2 Modules of the 3D printed flow system and the final assembled system

min). In this manner the student could easily explore the mixing ability of the reactor chip and retention times via addition or subtraction of one or more circular disk reactors (CDRs). Analysis via HPLC would also provide them with ready access to data whilst increasing the safety for the student as analysis focussed on differences in mixing rates rather than reactions (Fig. 3).

During initial experiments, we observed that the mixing of the two isopropanol solutions of $p$-cresol and 2-chlorophenol (differing viscosities) were travelling through the system at subtly different flow rates as calculated by HPLC (Fig. 4a). In order to demonstrate that this result was due to the viscosity and not the part of an interaction of one of the alcohols with the $3 \mathrm{D}$ printed reactors, we carried out a pre-mixing experiment, where the alcohols were injected into the same loop in a 1:1 manner, clearly demonstrating the effect of separate viscosities on the system (Fig. 4b).

Whilst isopropanol is a relatively viscous solvent used in continuous flow organic chemistry, the difference between two different solutions was evidently large enough to result in different flow rates. As a result of the fact that the narrow capillary flow resistor at the end of the flow path dictated the flow rate of the solution, the difference in flow rates of the two solutions at this position was thought to be responsible for the difference in flow rates and strongly suggested that the two solutions had not mixed sufficiently before this point. This was also clearly visible in a qualitative experiment where two coloured solutions were injected via loops A and B (Fig. 5). The opaque nature of the polypropylene (PP) mixing chip

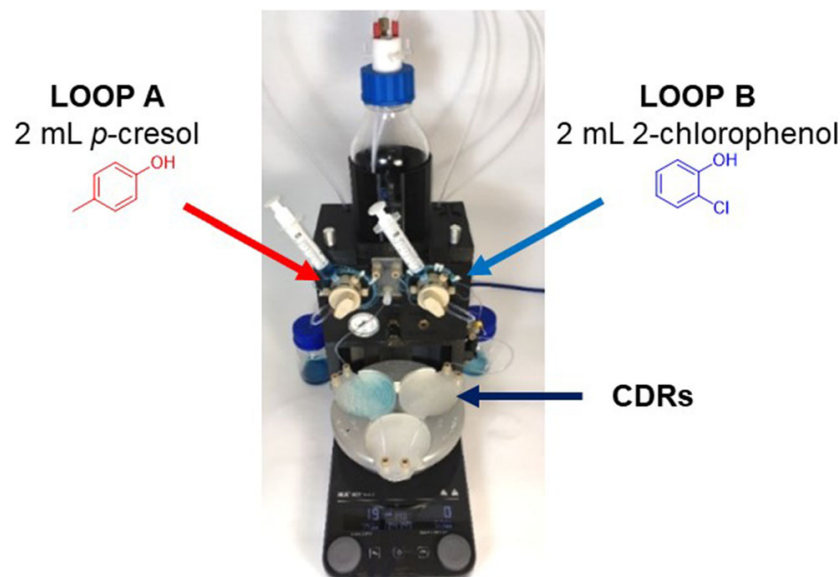

Fig. 3 The setup of the continuous flow system used for experiments

meant that the reduced mixing was clearly visible and could also be observed in the PP CDRs.

As shown in Fig. 5, laminar flow predominated in the designed poor mixing mesoscale mixing chip of this system. This meant that the poor mixing likely led to viscositystratified $[12,13]$ flow which could be responsible for the difference in the observed flow rates of a simple T-mixer. This could clearly have the potential to affect reaction stoichiometry and was therefore an important issue to address in the students' research. In order to determine whether this was an issue with the pressure driven nature of our system or was a more widespread problem within continuous flow chemistry equipment, we carried out the same mixing experiments on commercial systems and in syringe pumps. One system (A) was available in house, and another was one was carried out independently by chemists based in industry (B). In order to avoid commercial sensitivity, the names and systems are not disclosed in this report, but it is indicative of the problems of mixing in flow chemistry at low flow rates in general and raises important questions of reaction stoichiometry when stoichiometric mixing is assumed (Fig. 6).

In all cases, the two different alcohols showed poor mixing and non-ideal ratios. Experiments were repeated following calibration of the flow systems, but with analogous results (supplementary information), clearly illustrating that it is a mixing rather than a pump issue. Swapping of the two injectants to explore pump effects also demonstrated that it was not a failure or fault in one of the pumps. The mixing issue that we observed is likely to be greater in our pressure-driven system, however in a system using HPLC pumps at the low flow rates carried out in this experiment, we attributed the difference in the flow rates and mixing to pulsation from misaligned pump strokes. This raises an important point in continuous flow chemistry, as inefficient or poor mixing in the ratios of reactants at the point of mixing or in the flow path can lead to impurities or incorrect ratios of reactants. This is also an issue between experiments as the stroke paths of the HPLC based 

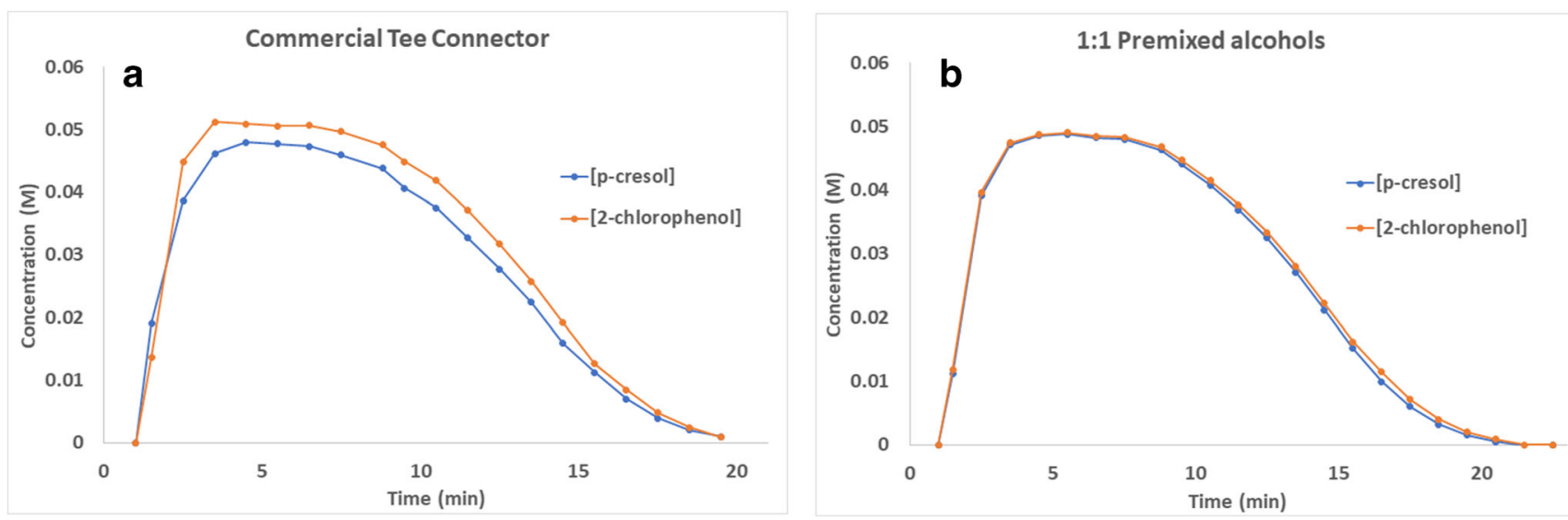

Fig. 4 a HPLC graph of the mixing and concentration profile of $p$-cresol and 2-chlorophenol via separate injection; $\mathbf{b}$ HPLC graph of the mixing and concentration profile of $p$-cresol and 2-chlorophenol via injection from the same loop

pumps can alter between reaction runs. The issue is less problematic in syringe-based systems, but is also a factor leading to imperfect mixing (Fig. 6c). In addition, flash flow chemistry, an area where flow chemistry excels over traditional methods $[14,15]$, requires the fast and accurate mixing of two reactants and so any mismatch in viscosities can lead to differing stoichiometries.

\section{Design and analysis of mixing chip efficiency for improved reactant stoichiometry}

In addition to understanding the basics of flow chemistry and given the initial mixing observations, the student was given the task of preparing a range of prototype mixing devices using FDM 3D printing to study the effect of mixing and laminar flow that could easily be incorporated into the mixing chip of the injection block of the flow reactor (Fig. 7).

The student was tasked with carrying out a brief survey of the literature to find simple architectures that could be quickly incorporated into a mixing device design and that could also be $3 \mathrm{D}$ printed in polypropylene. In order to evaluate different mixing devices, the student used Tinkercad $\AA$, an easily accessible free online drawing package. The student was able to quickly design a variety of mixing devices for evaluation (Fig. 8).

Initially, passive mixing devices were printed using an Ultimaker 3 with polypropylene filament. Devices A and B were used to investigate whether the angle of mixing was important as has been suggested [16]. Devices C and D were designed to investigate the effect of increasing turbulence within the channels of the device by incorporating baffles [17]. Following analysis, the T-shaped mixing point offered a small improvement in the ratio of the two phenols as expected [16]. It could be suggested that negative angled Y-type mixers could have been investigated as these have been shown to affect greater mixing efficiency [18]. The use of mixing device $\mathrm{C}$ showed another small improvement in mixing efficiency however the additional baffles and longer flow path of mixing device $\mathrm{D}$ resulted in a worse ratio. This was thought to be due to the increased likelihood of bubble formation and non-wetting of the channel surface [19] as well as unoptimized spacing of the baffles. It is also worth noting the increased dispersion in this device (Fig. 9).
Fig. 5 a Illustration of poor mixing in a specially designed mixing chip to show poor mixing; b PFA tubing connecting the mixing chip with the CDRs; $\mathbf{c}$ the output into the CRDs, highlighting that despite passing through tubing, the two colours are still not mixed
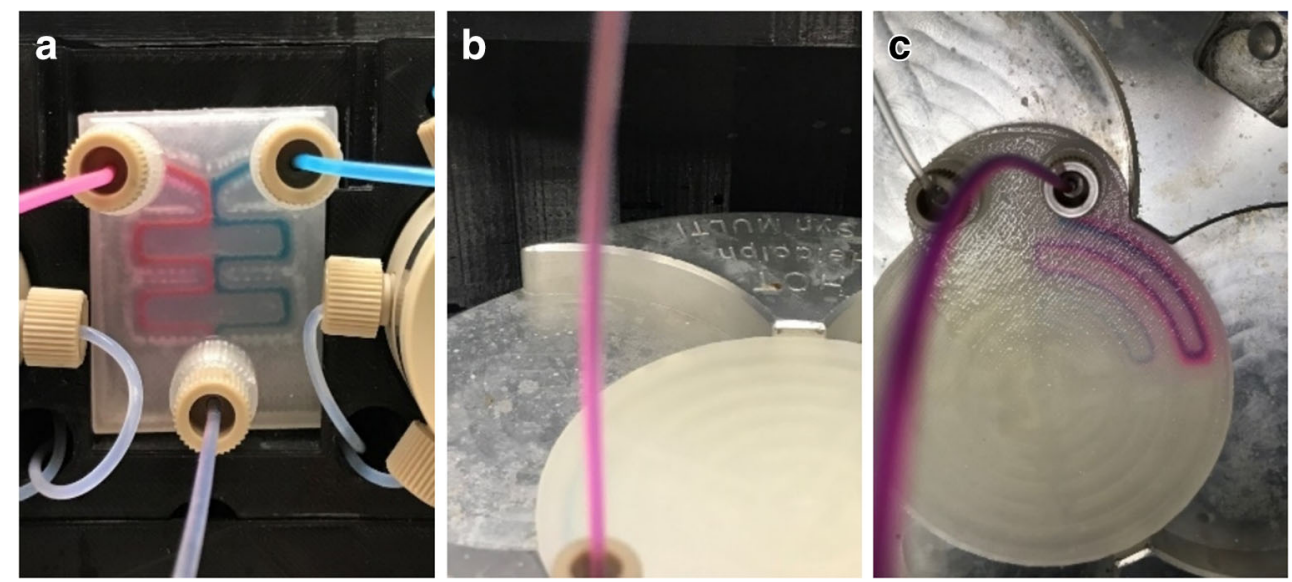


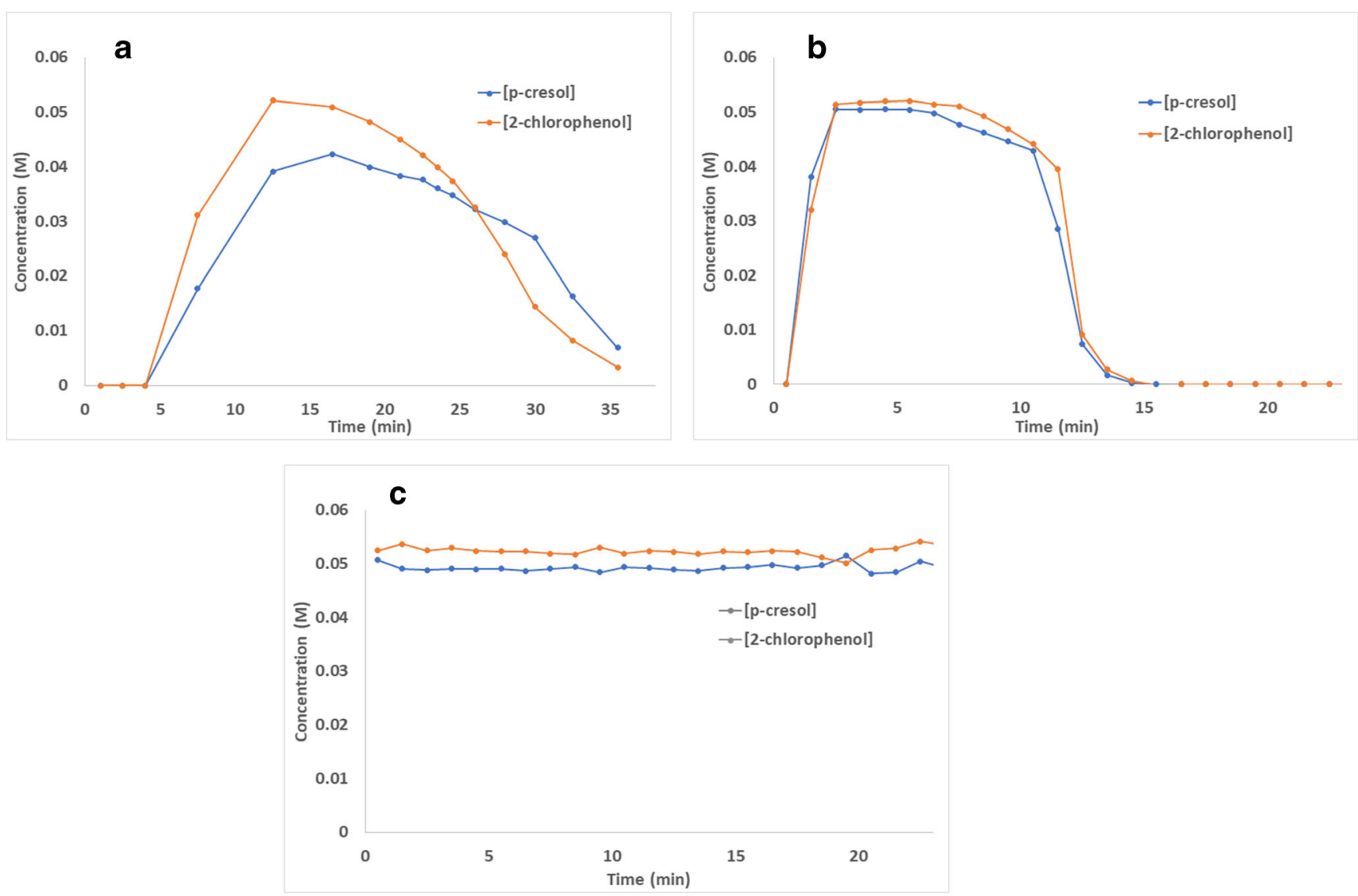

Fig. 6 a Commercial system 1 (internal) same protocol followed; b Commercial system 2 (external) same protocol followed; c Syringe pump - same protocol followed

\section{Design and development of an active mixing chip}

Given the relatively low improvements in mixing efficiency, the student designed and prepared an active mixing device which consisted of a chamber where a magnetic stir bar could be placed within the flow path. This was inspired by the relatively unknown miniature continuous flow reactor which was used for ultrafast nitration reactions [20,21]; more recent examples have shown utility in flow chemistry [22-24]. The mixing device would then be able to be used with a standard laboratory stirrer hotplate. Initially the mixing chamber was designed for a small Teflon coated cylindrical magnetic stir bar $(10 \times 3 \mathrm{~mm})$. In a subsequently design the height of the mixing chamber was modified to fit a cross-shape magnetic stir bar $(10 \times 5 \mathrm{~mm})$ (Fig. 10).

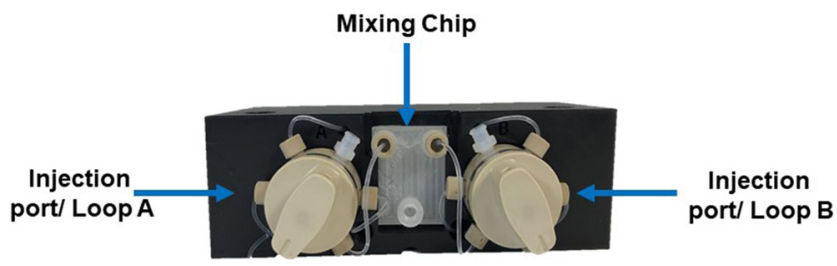

Fig. 7 Location of the mixing chip used for flow mixing experiments
In order to incorporate the stirrer bead within the 3D printed housing, the magnetic stir bar was placed into the chamber during the $3 \mathrm{D}$ print whilst paused and then printing continued to provide a sealed contiguous device. The curvature of the top of the mixing chamber was designed so that the print head would deposit filament in overlapping concentric circles to ensure the device was fully sealed (Fig. 11). In order to $3 \mathrm{D}$ print the desired mixing chip, a layer height of $0.15 \mathrm{~mm}$, infill of $100 \%$ and a flow rate of $110 \%$ was used.

When the two stirrer devices were placed on the stirrer hotplate and the rotation of the stirrer was set at $500 \mathrm{rpm}$, we were pleased to observe efficient mixing in both cases with the cylindrical stirrer and the crosshead stirrer. Whilst the smaller cylindrical stirrer improved mixing, it had a tendency to "catch" at higher speeds, whilst the larger one possessed improved speed stability leading to the perfect mixing of both alcohols as seen below (Fig. 12b). Increases in speed or reduction led to lower levels of mixing of the two reactants, with $500 \mathrm{rpm}$ representing the optimum speed (Supplementary Information).

The efficient mixing could also be observed qualitatively as seen via the injection and mixing of two different coloured solutions (Fig. 13), standing in stark contrast to that of the chip designed to have poor mixing (Fig. 4). 
Fig. 8 a Y-mixer chip; b T-mixer chip; c Simple baffle mixer chip; d Increased baffle chip
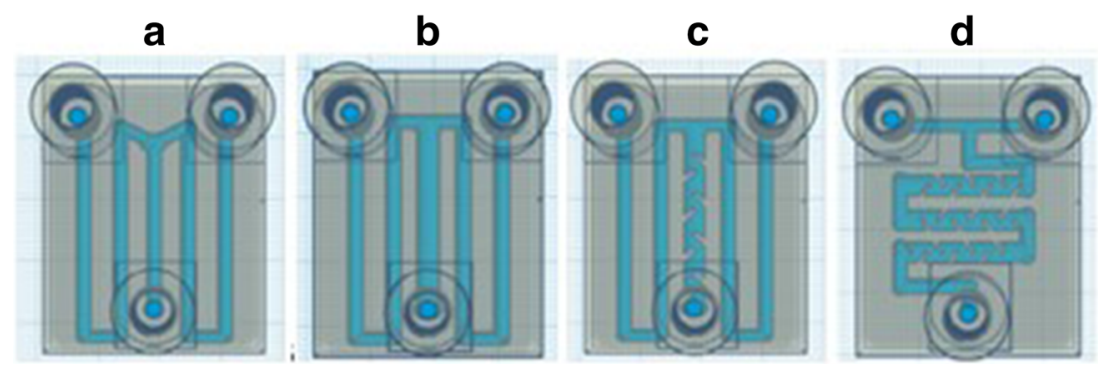
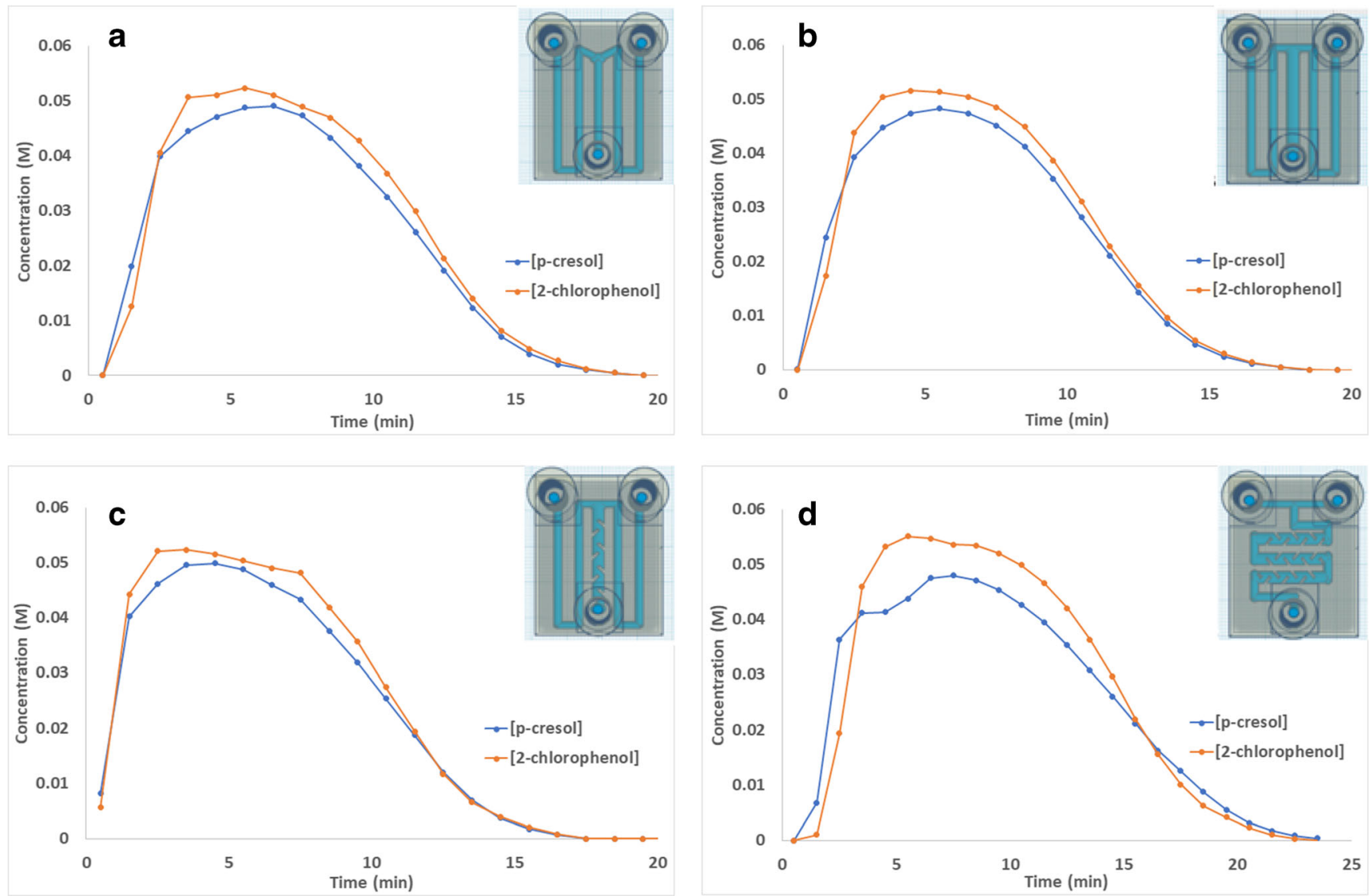

Fig. 9 a HPLC results showing Y-mixer chip mixing efficiency; b HPLC results showing T-mixer chip mixing efficiency; $\mathbf{c}$ HPLC results showing Simple baffle mixer chip mixing efficiency; $\mathbf{d}$ HPLC results showing Increased baffle chip mixing efficiency
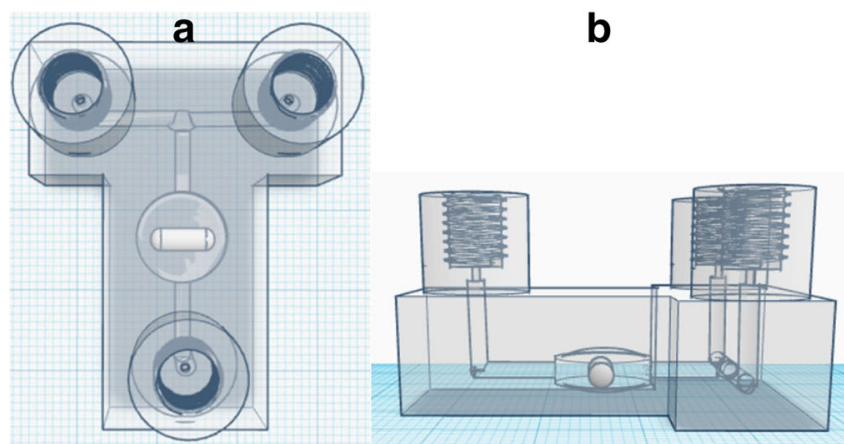

Fig. 10 a CAD design from the top face of the active mixer with a cylindrical stirrer bead; $\mathbf{b}$ CAD design from the side face of the active mixer with a cylindrical stirrer bead; c CAD design from the top face of
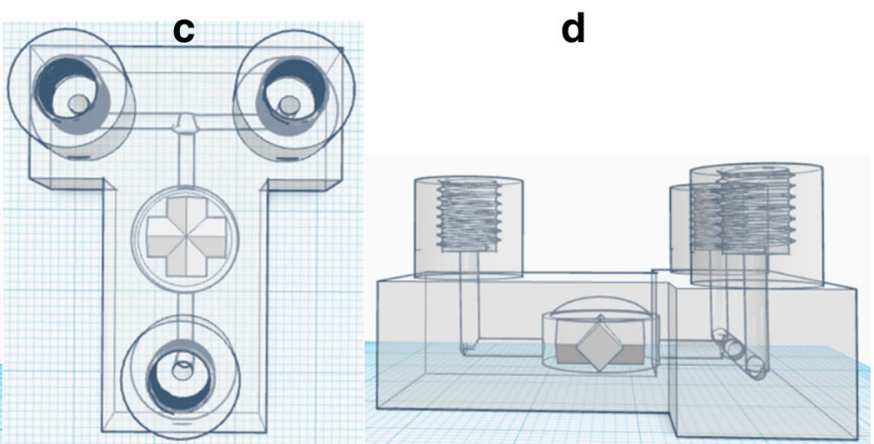

the active mixer with a crosshead stirrer bead; $\mathbf{d}$ CAD design from the side face of the active mixer with a crosshead stirrer bead 


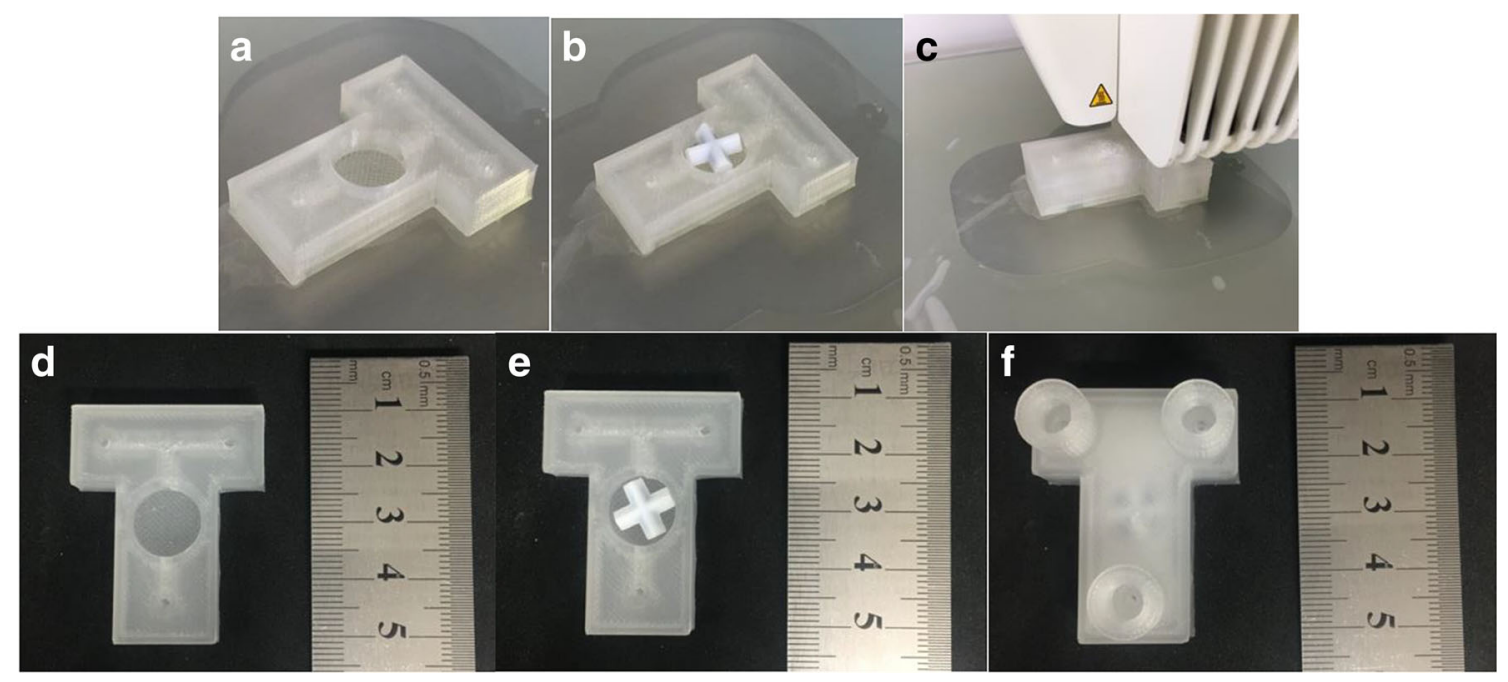

Fig. 11 a 3D Printing paused before addition of stirrer bead showing the central cavity; b Crosshead stirrer inserted into the cavity; c 3D Printing continued after insertion; $\mathbf{d}$ Size dimension of the mixer; e Size fit of the crosshead stirrer; $\mathbf{f}$ Fully 3D printed device
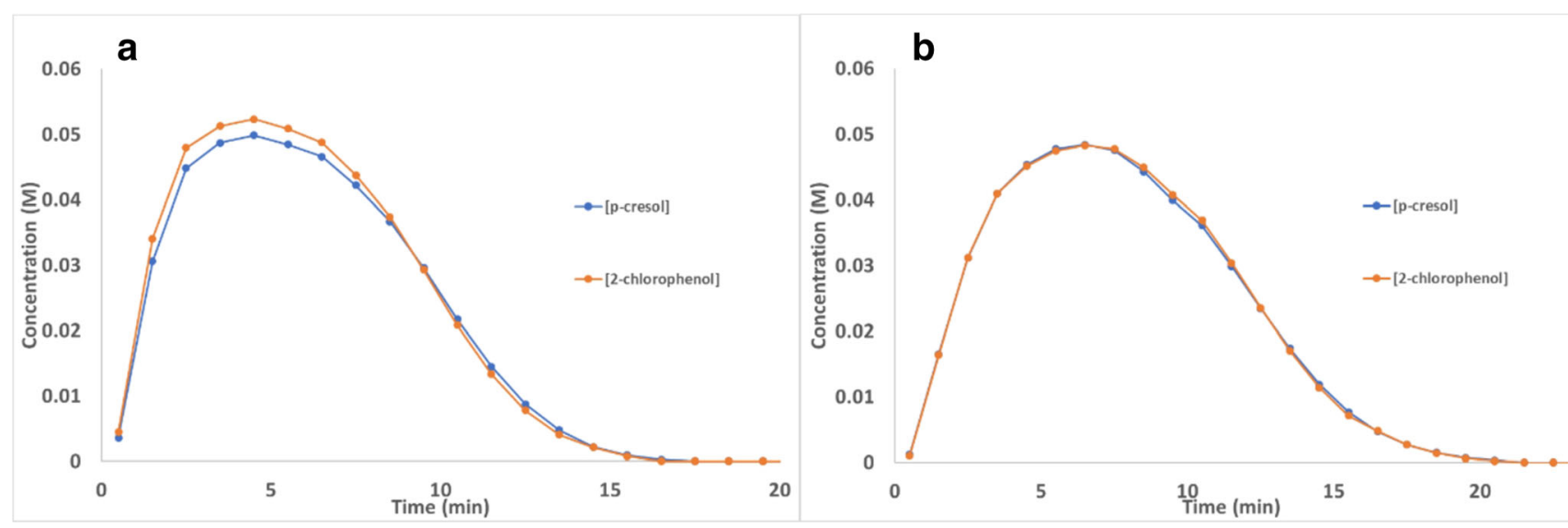

Fig. 12 a Graph of concentration versus time demonstrating the mixing efficiency of a cylindrical stirrer, mixed at 500 rpm; b Graph of concentration versus time demonstrating the mixing efficiency of a crosshead stirrer, mixed at $500 \mathrm{rpm}$

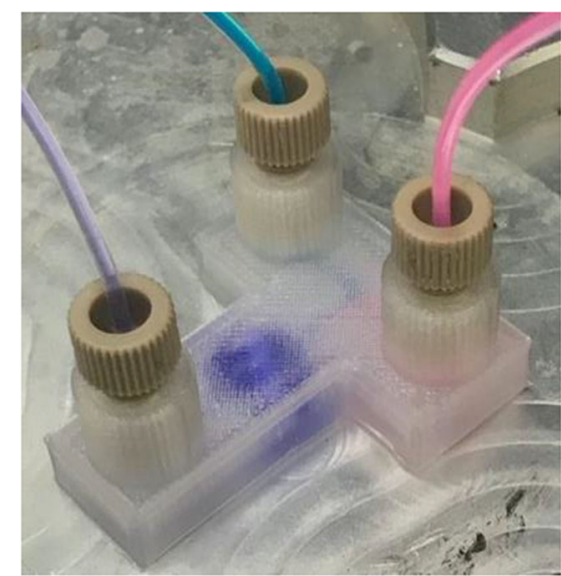

Fig. 13 Mixing of two different coloured dyes in the crosshead stirrer mixing chip with the speed of rotation of the stirrer hotplate set at $500 \mathrm{rpm}$
The results of the mixing experiments are summarised in Table 1 and it can clearly be seen that the mixing efficiency of the cross shaped stirrer bar is far greater than that of the passive mixing devices with the lower standard deviation indicative of better mixing efficiency (Table 1).

\section{Development of a single flow system with parallel reaction experimentation}

One of the key weaknesses of current continuous flow systems stems from their reliance on a single back pressure regulator. This clearly limits their potential as reactions can only be run in sequence. With an improvement in mixing achieved, we were intrigued by the possibility of modifying the system so that multiple reactions could be run in parallel on the same system as shown below (Fig. 14). As our system regulates the pressure by capillary resistors, we envisaged that it would be possible to 
Table 1 Mixing efficiency of mixer chips

\begin{tabular}{lll}
\hline Mixing Device & Average Standard Deviation (mM) & $\begin{array}{l}\text { Sum of Standard Deviation } \\
(\mathrm{mM})\end{array}$ \\
\hline Commercial Tee (PEEK) & 2.17 & 43.4 \\
Y-shape passive (PP) & 1.80 & 34.2 \\
T-shape passive (PP) & 1.75 & 33.2 \\
Short baffle & 1.61 & 27.3 \\
Long baffle & 3.45 & 82.9 \\
Cylindrical stir bar & 0.992 & 16.9 \\
Cross-shape stir bar & $\mathbf{0 . 1 9 4}$ & $\mathbf{4 . 0 8}$ \\
\hline
\end{tabular}

Fig. 14 Illustration of the flow arrangement for parallel reactions and actual realised experimental setup

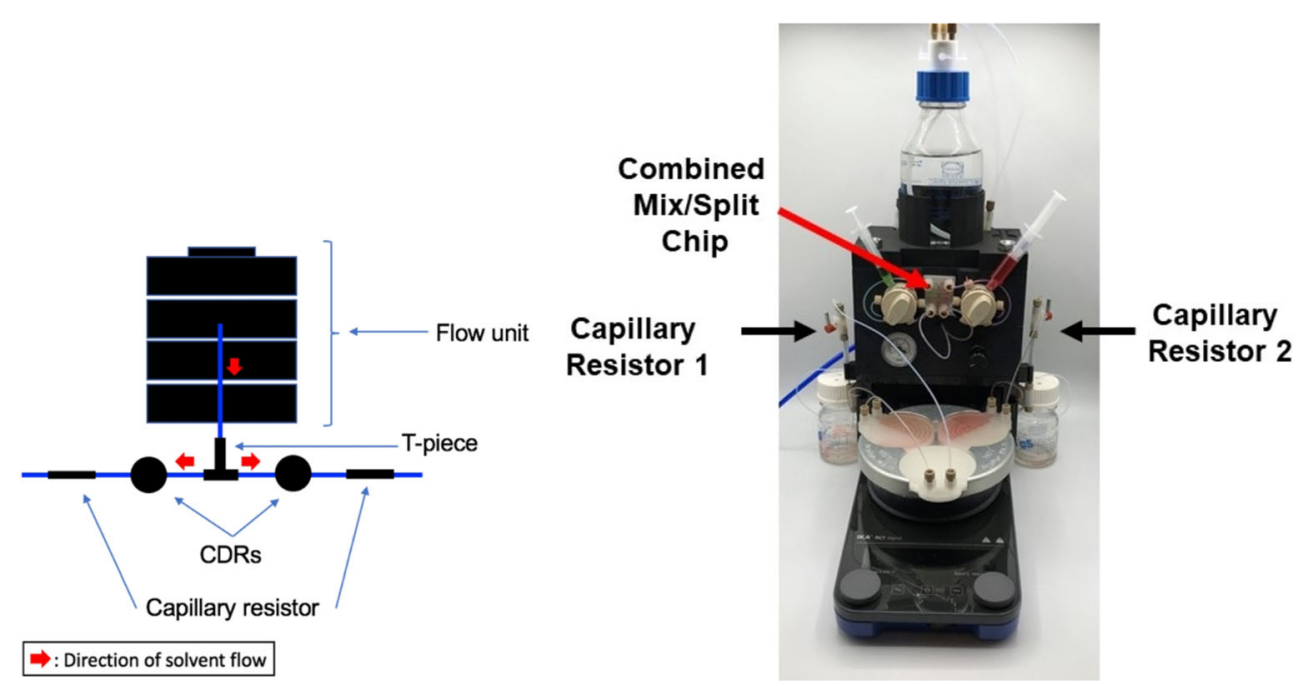

run two (or more) reactions in parallel via splitting after the mixing chip and running the two flows through two differing paths from a single input. The addition of the same capillary resistor after each would then enable direct comparison between experiments with no temperature variation as the same stirrer hotplate can be used. Variation in residence time and or flow path is an obvious possibility with this approach, and also via the use of two hotplates, variation in temperature (Fig. 14).

In order to explore the potential of running multiple reactions at the same time by splitting the output of the active mixing device between 2 identical capillary resistors on the same stirrer hotplate (Fig. 14), we compared the mixing
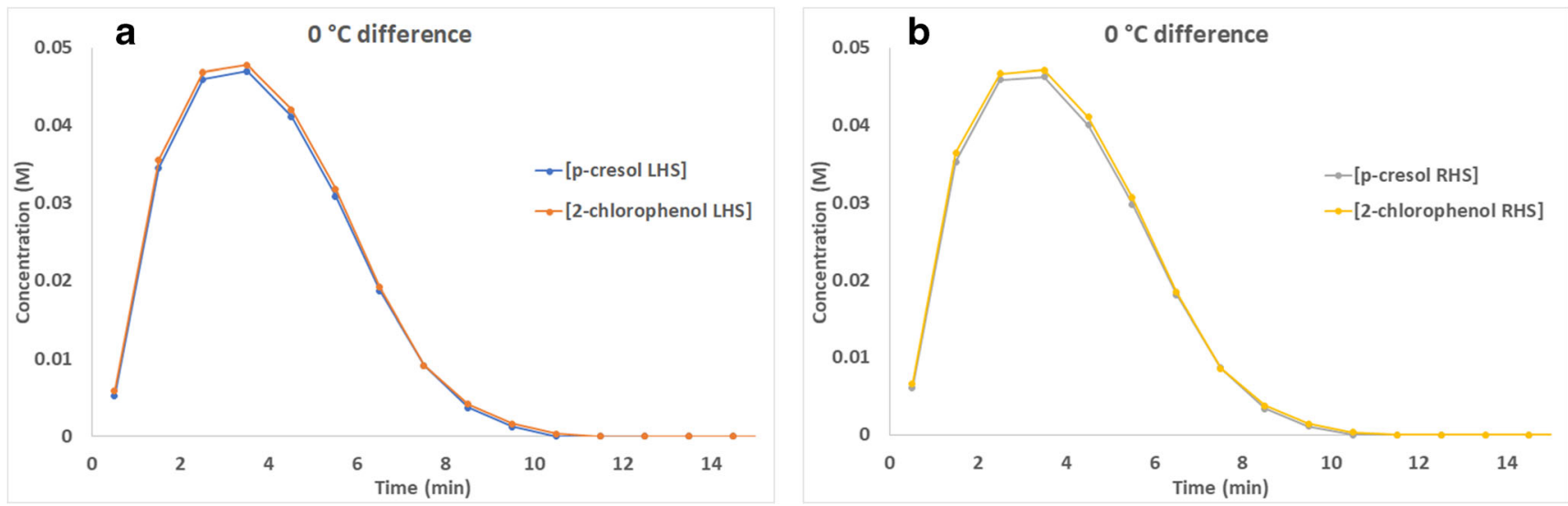

Fig. 15 a Graph of concentration versus time demonstrating the mixing efficiency of the Left-Sided capillary; $\mathbf{b}$ Graph of concentration versus time demonstrating the mixing efficiency of the Right-Sided capillary 


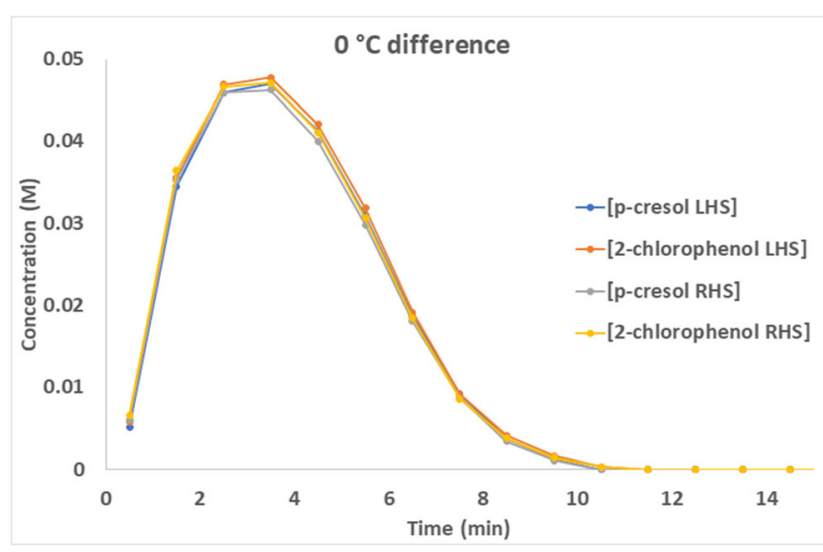

Fig. 16 Combined graphs from Fig. 15 of concentration versus time demonstrating the mixing efficiency of the both the Left- and RightSided capillary experiments

profiles after splitting of the two reactor paths via a single CDR (Fig. 15).

As can be seen from the combined graphs, the mixing of the two sides of the flow path were perfectly mixed, demonstrating that reactions could easily be run in parallel with optimal mixing and so would provide ideal information from two reactions (Fig. 16).
The next experiment then looked at the mixing profiles from analysis of two different CDRs. The standard one possessing an internal volume of $2.68 \mathrm{~mL}$ and a narrower channelled modified CDR with an internal volume of $4.03 \mathrm{~mL}$. As can be seen from the graph of concentration versus time, the different flow paths lead to differing concentration profiles, clearly illustrating the potential of this approach. The picture of the two paths is illustrative with coloured dyes to highlight the concept used in this experiment (Fig. 17).

Following demonstration of the proof of concept, the student next focussed on a comparison of one versus two CDRs on the reaction profile. This therefore compared a reactor volume of $2.68 \mathrm{~mL}$ versus $5.36 \mathrm{~mL}$ in the combined system (Fig. 18).

As can be seen, the addition of a second reactor led to an expected slightly more diffuse peak profile of the material that was passed through the two combined CDRs with a greater overall internal volume. Pleasingly, this clearly demonstrates that experiments can easily be run in parallel with differing flow paths and path lengths via addition or subtraction of CDRs.
Fig. 17 a Illustrative picture of the two differing flow paths used in the experiment with a normal CDR and modified narrower flow path CDR on the right; $\mathbf{b}$ Graph of concentration versus time profile of the normal CDR versus the narrow channel CDR
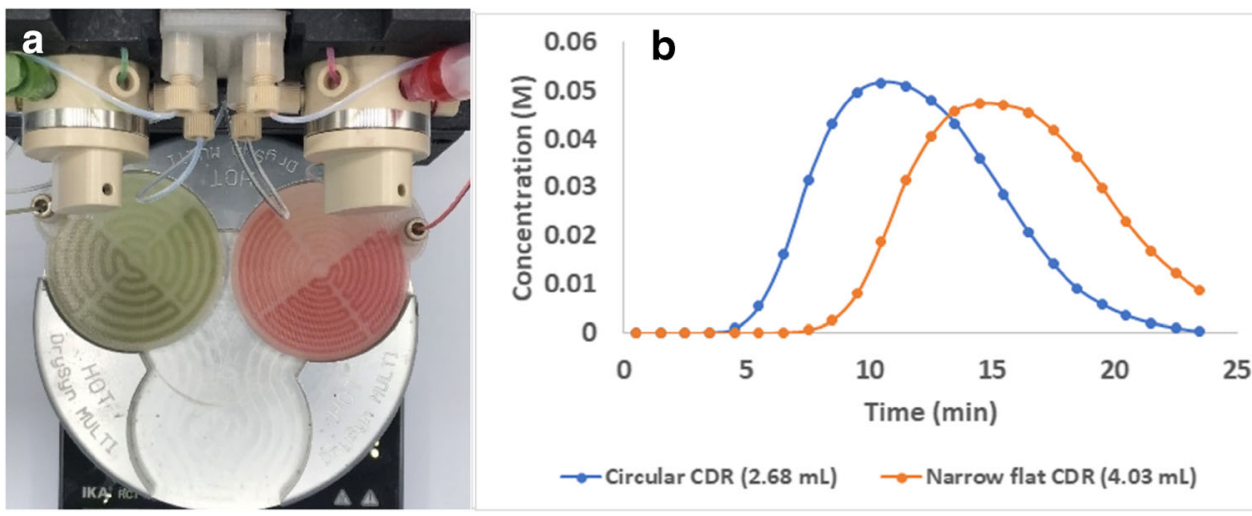

$\multimap$ Circular CDR $(2.68 \mathrm{~mL}) \quad-$ Narrow flat CDR $(4.03 \mathrm{~mL})$
Fig. 18 a Illustrative picture of the two differing flow paths used in the experiment with two CDRs versus a single CDR; $\mathbf{b}$ Graph of concentration versus time profile of the single CDR versus two CDRs with a longer retention time
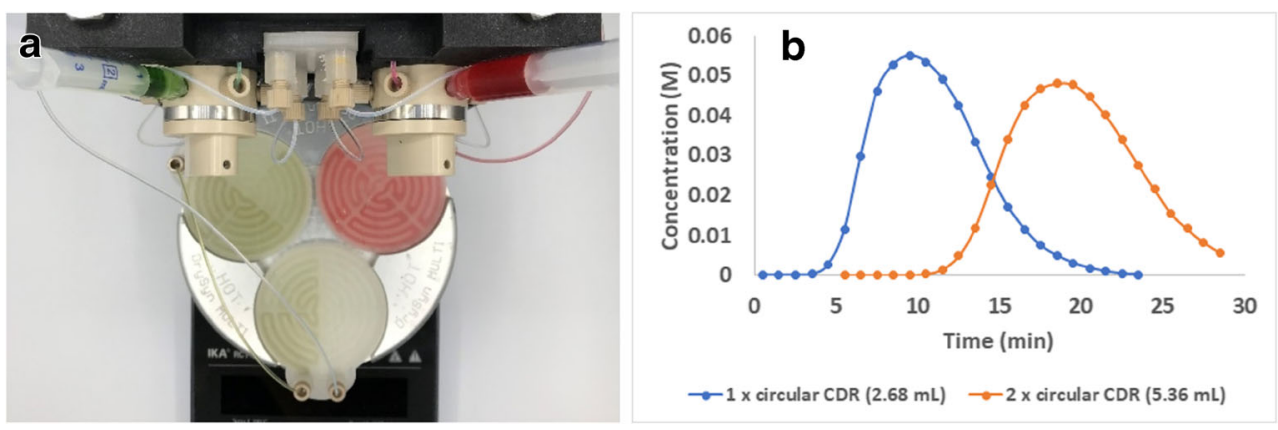


\section{Conclusions}

In this report, we have demonstrated that the teaching of practical flow chemistry to an undergraduate student (N.T.) is clearly possible at low cost as part of a short research project (4 months). Whilst it is obvious that not all projects would lead to such an outcome from such a student, it highlights what is possible with continuous flow at an undergraduate level. In being able to assemble the equipment, the student was able to gain a practical understanding of flow chemistry and applied this to solve a mixing issue due to laminar flow. This clearly raises the prospect of carrying out some practicals detailed herein with undergraduate students due to their simplicity and visual aspect which provides a ready visualisation of flow chemistry with a system that is relatively low in cost due to its $3 \mathrm{D}$ printed nature. In addition, the student was also able to develop the concept further via splitting after active mixing to demonstrate that more excitingly, flow chemistry reactions can also be run in parallel from a single reaction input and on a single continuous flow system. This clear reduction in reaction timing and improvement in reaction efficiency, will enable additional multiple parallel experiments to be run not only by undergraduate students, but also on a research-based level. The use of $3 \mathrm{D}$ printing to develop both the mixing chips and the CDRs raises the prospect of vastly reduced costs of undergraduate practical chemistry as the reactors used in this study cost less than $\$ 1$ to produce.

Funding The authors acknowledge funding from Zurich Insurance for the work carried out in this study which was given to fund MRP.

Open Access This article is licensed under a Creative Commons Attribution 4.0 International License, which permits use, sharing, adaptation, distribution and reproduction in any medium or format, as long as you give appropriate credit to the original author(s) and the source, provide a link to the Creative Commons licence, and indicate if changes were made. The images or other third party material in this article are included in the article's Creative Commons licence, unless indicated otherwise in a credit line to the material. If material is not included in the article's Creative Commons licence and your intended use is not permitted by statutory regulation or exceeds the permitted use, you will need to obtain permission directly from the copyright holder. To view a copy of this licence, visit http://creativecommons.org/licenses/by/4.0/.

\section{References}

1. Mullin R (2019) Off the drawing board. Chemical \& Engineering News, Apr 28, 2019, 97, 17 https://cen.acs.org/pharmaceuticals/ Off-drawing-board/97/i17. Accessed 28/08/2020
2. König B, Kreitmeier P, Hilgers P, Wirth T (2013) Flow chemistry in undergraduate organic chemistry education. J Chem Educ 90: 934-936

3. Tabassum T, Iloska M, Scuereb D, Taira N, Jin C, Zaitsev V, Afshar F, Kim T (2018) Development and application of 3D printed mesoreactors in chemical engineering education. J Chem Educ 95: 783-790

4. Leibfarth FA, Russell MG, Langley DM, Seo H, Kelly LP, Carney DW, Sello JK, Jamison TF (2018) Continuous-flow chemistry in undergraduate education: sustainable conversion of reclaimed vegetable oil into biodiesel. J Chem Educ 95: 1371-1375

5. Neumaier JM, Madani A, Klein T, Ziegler T (2019) Low-budget 3D-printed equipment for continuous flow reactions. Beilstein $\mathrm{J}$ Org Chem 15:558-566

6. Penny MR, Rao ZX, Peniche BF, Hilton ST (2019) Modular 3D printed compressed air driven continuous-flow systems for chemical synthesis. Eur J Org Chem 2019:3783-3787

7. Rao ZX, Patel B, Monaco A, Cao ZJ, Barniol-Xicota M, Pichon E, Ladlow M, Hilton ST (2017) 3D-printed polypropylene continuous-flow column reactors: exploration of reactor utility in snar reactions and the synthesis of bicyclic and tetracyclic heterocycles. Eur J Org Chem 2017:6499-6504

8. Penny MR, Hilton ST (2020) Design and development of 3D printed catalytically active stirrers for chemical synthesis. React Chem Eng 5:853-858

9. Penny MR, Rao ZX, Ishaq A, Thavarajah R, Hilton S (2020) 3D printed tetrakis(triphenylphosphine)palladium (0) impregnated stirrer devices for suzuki-miyaura cross-coupling reactions. ChemRxiv Preprint. https://doi.org/10. 26434/chemrxiv.12798545.v1

10. Fekete M, Glasnov T (2014) Technology overview/Overview of the devices. In: Darvas F, Hessel V, Dorman G (eds) Flow Chemistry. Fundamentals, vol 1. De Gruyter, Berlin, pp $95-140$

11. Vangunten MT, Walker UJ, Do HG, Knust KN (2020) 3D-printed microfluidics for hands-on undergraduate laboratory experiments. J Chem Educ 97:178-183

12. Truzzolillo D, Cipelletti L (2018) Hydrodynamic instabilities in miscible fluids. J Phys: Condens Matter 30:033001

13. Das S, Gada VH, Sharma A (2015) Analytical and level set methodbased numerical study for two-phase stratified flow in a pipe. Numer Heat Transf Part A 67:1253-1281

14. Yoshida J-I, Kim H, Nagaki A (2017) "Impossible" chemistries based on flow and micro. J Flow Chem 7:60-64

15. Gutmann B, Kappe CO (2017) Forbidden chemistries - Paths to a sustainable future engaging continuous processing. J Flow Chem 7: 65-71

16. Gobby D, Angeli P, Gavriilidis A (2001) Mixing characteristics of T-type microfluidic mixers. J Micromech Microeng 11:126

17. Al-Atabi M (2011) Design and assessment of a novel static mixer. ï) CCan J Chem Eng 89:550-554

18. Hsieh S-S, Lin J-W, Chen J-H (2013) Mixing efficiency of Y-type micromixers with different angles. Int J Heat Fluid Flow 44:130 139

19. Pereiro I, Fomitcheva Khartchenko A, Petrini L, Kaigala GV (2019) Nip the bubble in the bud: a guide to avoid gas nucleation in microfluidics Lab. Chip 19:2296-2314

20. Brennecke HM, Kobe KA (1956) Mixed acid nitration of toluene. Ind Eng Chem 48:1298-1304

21. Cantillo D (2015) A pioneering early microreactor concept for ultrafast nitration reactions - Placing the seminal Brennecke and Kobe 1956 contribution into perspective. J Flow Chem 5:195-196 
22. Scotti G, Nilsson SME, Haapala M, Pöhö P, af Gennäs GB, Yli-Kauhaluoma J, Kotiaho T (2017) A miniaturised 3D printed polypropylene reactor for online reaction analysis by mass spectrometry. React Chem Eng 2:299-303

23. Chapman MR, Kwan MHT, King G, Jolley KE, Hussain M, Hussain S, Salama IE, González Niño C, Thompson LA, Bayana ME, Clayton AD, Nguyen BN, Turner NJ, Kapur N, Blacker AJ (2017) Simple and versatile laboratory scale CSTR for multiphasic continuous-flow chemistry and long residence times. Org Process Res Dev 21:1294-1301

24. Mensing GA, Pearce TM, Graham MD, Beebe DJ (2004) An externally driven magnetic microstirrer. Phil Trans R Soc Lond A 362:1059-1068

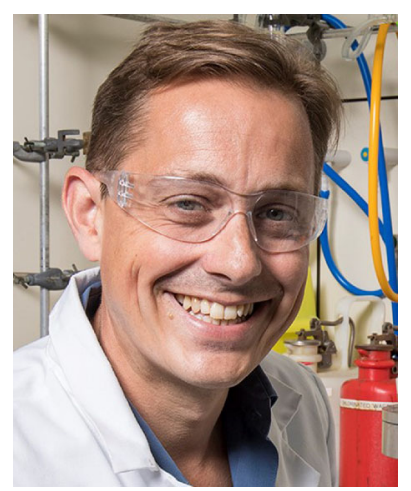

Dr Stephen Hilton is an Associate Professor at UCL school of Pharmacy, having previously worked as an academic fellow at the School of Pharmacy, University of London. He carried out postdoctoral research at the Institute of Cancer Research (ICR) and with Professor William Motherwell at UCL. His $\mathrm{PhD}$ at Kingston University under Professor Keith Jones focussed on radical chemical approaches to indole alkaloids. Dr Hilton's diverse research interests range from medicinal chemistry, scale-up synthesis and new technology with an emphasis on the applications of $3 \mathrm{D}$ printing in synthetic chemistry and Pharmaceutical applications. 